\title{
Dysphagia Prevalence, Time Course, and Association with Probable Sarcopenia, Inactivity, Malnutrition, and Disease Status in Older Patients Admitted to an Emergency Department: A Secondary Analysis of Cohort Study Data
}

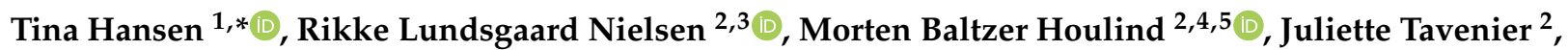
Line Jee Hartmann Rasmussen 2,6 (D), Lillian Mørch Jørgensen 2,7, Charlotte Treldal 2,4,5, Anne Marie Beck 8,9, Mette Merete Pedersen ${ }^{2,3}\left(\mathbb{D}\right.$, Ove Andersen ${ }^{2,3,7}$, Janne Petersen $2,10,11\left(\mathbb{D}\right.$ and Aino Leegaard Andersen ${ }^{2,3}(\mathbb{D}$

1 Department of Occupational Therapy and Physiotherapy, Copenhagen University Hospital Amager and Hvidovre, 2650 Hvidovre, Denmark

2 Department of Clinical Research, Copenhagen University Hospital Amager and Hvidovre, 2650 Hvidovre, Denmark; rikke.lundsgaard.nielsen@regionh.dk (R.L.N.); morten.baltzer.houlind@regionh.dk (M.B.H.); juliette.tavenier@regionh.dk (J.T.); line.jee.hartmann.rasmussen@regionh.dk (L.J.H.R.); lillian.moerch.joergensen@regionh.dk (L.M.J.); ctreldal@gmail.com (C.T.); mette.merete.pedersen@regionh.dk (M.M.P.); ove.andersen@regionh.dk (O.A.); janne.petersen.01@regionh.dk (J.P.); aino.leegaard.andersen@regionh.dk (A.L.A.)

check for updates

Citation: Hansen, T.; Nielsen, R.L.; Houlind, M.B.; Tavenier, J.;

Rasmussen, L.J.H.; Jørgensen, L.M.; Treldal, C.; Beck, A.M.; Pedersen, M.M.; Andersen, O.; et al. Dysphagia Prevalence, Time Course, and Association with Probable Sarcopenia, Inactivity, Malnutrition, and Disease Status in Older Patients Admitted to an Emergency Department: A Secondary Analysis of Cohort Study Data. Geriatrics 2021, 6, 46. https:// doi.org/10.3390/geriatrics6020046

Academic Editor: David G. Smithard

Received: 7 April 2021

Accepted: 23 April 2021

Published: 26 April 2021

Publisher's Note: MDPI stays neutra with regard to jurisdictional claims in published maps and institutional affiliations.

Copyright: (C) 2021 by the authors. Licensee MDPI, Basel, Switzerland. This article is an open access article distributed under the terms and conditions of the Creative Commons Attribution (CC BY) license (https:// creativecommons.org/licenses/by/ $4.0 /)$.
3 Department of Clinical Medicine, Faculty of Health and Medical Sciences, University of Copenhagen, Blegdamsvej 3B, 2200 Copenhagen N, Denmark

4 The Capital Region Pharmacy, Marielundsvej 25, 2730 Herlev, Denmark

5 Department of Drug Design and Pharmacology, University of Copenhagen, Universitetsparken 2, 2100 Copenhagen $\varnothing$, Denmark

6 Department of Psychology and Neuroscience, Duke University, 2020 W Main St, Durham, NC 27705, USA

7 Emergency Department, Copenhagen University Hospital Amager and Hvidovre, Kettegaards alle 30, 2650 Hvidovre, Denmark

8 Department of Nursing and Nutrition, University College Copenhagen, Sigurdsgade 26, 2200 Copenhagen N, Denmark; Anne.Marie.Beck@regionh.dk

9 Dietetic and Nutritional Research Unit, Herlev-Gentofte University Hospital, Borgmester Ib Juuls Vej 50, 2730 Herlev, Denmark

10 Center of Clinical Research and Prevention and Department of Clinical Pharmacology, Copenhagen University Hospital Bispebjerg and Frederiksberg, Nordre Fasanvej 57, 2000 Frederiksberg, Denmark

11 Section of Biostatistics, Department of Public Health, University of Copenhagen, Øster Farimagsgade 5, 1014 Copenhagen K, Denmark

* Correspondence: tina.hansen.18@regionh.dk; Tel.: +45-29243586

Abstract: There is evolving evidence for an association between dysphagia and sarcopenia in older adults. For optimizing the acute health care initiative across health care settings, this study investigated prevalence and time-course of dysphagia in older patients admitted to an emergency department (ED) as well as its association with parameters for probable sarcopenia, inactivity, malnutrition, disease status, and systemic inflammation. A secondary analysis of data from the FAM-CPH cohort study on acutely admitted older medical patients $(n=125)$. Data were collected upon ED admission as well as four and 56 weeks after discharge. Using the Eating Assessment Tool cut-off score $\geq 2$, signs of dysphagia were present in $34 \%$ of the patients at ED admission and persisted in $25 \%$ of the patients 56 weeks after discharge. Signs of dysphagia at 56-week follow-up were significantly $(p<0.05)$ associated with probable sarcopenia (low handgrip strength $(\mathrm{OR}=3.79)$, low leg muscle strength $(\mathrm{OR}=8.14)$, and low physical performance $(\mathrm{OR}=5.68)$ ) and with baseline swallowing inactivity $(\mathrm{OR}=5.61)$, malnutrition $(\mathrm{OR}=4.35)$, and systemic inflammation $(\mathrm{OR}=1.33)$. Signs of dysphagia in older patients admitted to an ED was prevalent, persisted 56 weeks after discharge, and was associated with probable sarcopenia and related conditions; all modifiable targets for management of dysphagia in older patients.

Keywords: swallowing difficulties; sarcopenia; inactivity; malnutrition; acute care; geriatric patients 


\section{Introduction}

Dysphagia in older adults is a frequent and serious condition that impairs swallowing efficiency and safety with increased risk of diminished nutritional intake and aspiration of foods and liquids [1,2]. Consequently, dysphagia is associated with aspiration pneumonia, malnutrition, weight loss, frequent hospital admissions with prolonged length of stay (LOS), increased mortality, decreased quality of life, as well as increased healthcare costs [1,3-7]. The prevalence of dysphagia in geriatric patients is reported as high as $50 \%$ [5-7] and can be caused by a range of diseases of the central and peripheral nervous system, structural, and mechanical changes of the aerodigestive tract as well as by advanced age $[1,2,8]$.

There is evolving evidence for an association between sarcopenia (low muscle strength, low muscle quantity or quality, and low physical performance) [9] and dysphagia in older adults $[8,10,11]$. Concurrent dysphagia and sarcopenia are observed in $6 \%$ of communitydwelling elders [12], in 13-30\% of geriatric inpatients [13-15], and in $32 \%$ of patients requiring dysphagia rehabilitation [16]. Sarcopenia of the whole body and swallowing muscles can be primary due to aging per se, or secondary in the event of physical inactivity, malnutrition, and/or systemic disease and inflammation $[8,11,17]$. The presence of concurrent dysphagia and sarcopenia is complex and there may be a two-way causal relationship between them generating detrimental synergies [8,11]. In a large retrospective cohort study, Maeda et al. [18] found that in older patients with normal swallow at admission, the risk of developing dysphagia during hospitalization was associated with indicators on sarcopenia, swallowing inactivity caused by diet restrictions with no oral intake or being on a texture modified diet, low performance status and immobilization, and low nutritional status. Likewise, dysphagia leads to reduced swallowing activity and malnutrition due to decreased food intake, which might induce or exacerbate sarcopenia [11].

Research on dysphagia and sarcopenia is still in its infancy and further research in various settings is needed $[8,11]$. During the last decades, the Danish healthcare system has been reorganized and centralized into fewer hospitals and a single-entry point through the emergency department (ED) with the aim to support cooperation across medical specialties and to contribute to faster treatment, reduced LOS and/or avoidance of unnecessary hospitalization [19]. In addition, the number of ED contacts and the proportion of contacts lasting $<24 \mathrm{~h}$ among the older population have increased, and a growing part of healthcare, treatment, and rehabilitation services are provided by the municipalities in the primary care setting [20]. Accordingly, it is recommended that the overall acute health care initiative is seen as a unified effort across sectors to ensure high quality and efficiency in the process for the acute older patient [20]. In this context, evidence of dysphagia prevalence, course, and association with sarcopenia in older patients admitted to the ED will contribute to optimizing patient trajectory from secondary health- to primary health- and social care settings. One Danish feasibility study reports a dysphagia prevalence of $24 \%$ in older patients admitted to the ED [21]. However, this study did not explicitly address the course of dysphagia or its association with sarcopenia. Therefore, the aim of the present study was to investigate the prevalence and time course of dysphagia and its association with parameters of sarcopenia, inactivity, malnutrition, disease status, and systemic inflammation in older patients acutely admitted to an ED within the Danish health care system.

\section{Materials and Methods}

\subsection{Design and Participants}

This study is a secondary analysis of data collected for a prospective, longitudinal observational study (the FAM-CPH cohort) investigating mechanisms for chronic inflammation and biological aging, risk of malnutrition, and unnecessary medication after admission to an ED at Copenhagen University Hospital, Hvidovre, Denmark (Clinical Trials.gov identifier: NCT03052192). Details on the FAM-CPH study have previously been 
described [22,23]. In short, eligible patients were aged $\geq 65$ years, Caucasian, and had sufficient Danish language skills. Patients were excluded if unable to participate due to cognitive impairments, terminally ill, or in need of isolation due to infectious disease. Data were collected from November 2016 to September 2018 upon ED admission, at 4 weeks and 56 weeks after discharge according to a comprehensive data collection manual.

\subsection{Data Included in the Secondary Analysis}

For the present secondary analysis, data were provided from the Research Electronic Capture tool (REDCap) [24] and included information on baseline patient characteristics, dysphagia (primary outcome), and a set of secondary outcomes related to sarcopenia, activity status, nutritional status, and disease status upon ED-admission (baseline), and at 4-week and 56-week follow-up after discharge.

\subsubsection{Patient Characteristics}

Patient characteristics at baseline includes demographics, admission diagnoses, LOS, and cognitive status assessed with the Orientation Memory Concentration-test (OMC) that provides a weighted total score ranging from $0-28$ points, where scores $\leq 17$ indicates moderate to severe cognitive impairment [25].

\subsubsection{Dysphagia}

Dysphagia was assessed by the Eating Assessment Tool (EAT-10); a patient-reported outcome measure of self-perceived dysphagia severity. The EAT-10 covers ten items, each reflecting a possible sign of dysphagia. The items are rated on a five-point response scale from 0 (no problem) to 4 (severe problem) with a total score ranging from 0-40 [26]. A total score $\geq 2$ has been shown as indicative of dysphagia with a sensitivity of $94.0 \%$ and a specificity of $70.9 \%$, when using video fluoroscopic swallowing examination as reference test [27]. This cut-off was used for estimating the prevalence of signs of dysphagia at the three time points. In addition, the course of dysphagia from baseline to 4-week and 56-week follow-up was categorized as follows: dysphagia persistently absent (EAT-10 total score $<2$ at all three time points); dysphagia persistently present (EAT-10 total score $\geq 2$ at all three time points); dysphagia remission (EAT-10 total score $\geq 2$ at baseline or at 4-week follow-up and EAT-10 total score $<2$ at 4-week or at 56-week follow-up), and dysphagia incident (EAT-10 total score $<2$ at baseline or at 4-week follow-up and EAT-10 total score $\geq 2$ at 4 -week or at 56-week follow-up).

\subsubsection{Sarcopenia}

In the FAM-CPH dataset, available sarcopenia-related parameters were muscle strength and physical performance. According to the European Working Group on Sarcopenia in Older People revised guidelines (EWGSOP2), low muscle strength indicates probable sarcopenia and physical performance provides information on sarcopenia severity [9].

Muscle strength was determined by handgrip strength (HGS) and leg strength and endurance. HGS was assessed using a digital hand-held dynamometer (model Digi-II, Saehan Corp., Masan, South Korea). Bedridden patients were assessed in the bed with an elevated backrest, and mobile patients were sitting on a chair with the elbow flexed at $90^{\circ}$ and the wrist in a neutral position [28]. The highest value of three consecutive attempts with the dominant hand is used for analyses. If the third trial elicited the highest value, additional trials were performed until maximum HGS was identified. Leg strength and endurance was assessed using the 30 second chair stand test (30-CST), which assesses how many times a patient can rise and sit from a standardized chair with the arms folded across the chest in 30 seconds. Only full standing positions are counted, and scores can range from 0 for those who cannot complete 1 stand to above 20 for more fit individuals [29]. Before data collection, patients were asked to do one or two practice repetitions to ensure they understood the expected performance. Physical performance was determined by the 
4-m gait speed (4MGS) test, of which, the fastest time of two trials (expressed in meter per second $(\mathrm{m} / \mathrm{s}))$ at usual pace was used for the analyses [30].

According to suggested cut-off points, the following thresholds were used for the sarcopenia-related parameters: HGS $<27 \mathrm{~kg}$ for men and $<16 \mathrm{~kg}$ for women, $30-\mathrm{CST}<9$ rises, and gait speed $\leq 0.8 \mathrm{~m} / \mathrm{s}[9,31]$. In the FAM-CPH dataset, information on muscle quality and quantity was not provided. According to the EWGSOP2 [9], a confirmed diagnosis of sarcopenia includes evidence of low muscle quality and quantity. Therefore, a definite diagnosis of sarcopenia as defined by EWGSOP2 [9] was not possible, and the three included sarcopenia parameters were considered individually as signs of probable sarcopenia.

\subsubsection{Activity, Nutritional, and Disease Status}

Activity status was represented by swallowing activity and functional performance status. Swallowing activity was determined on basis of self-report using the Simplified Nutritional Appetite Questionnaire (SNAQ) with four items to be rated on a five-point Likert scale providing a total score from 4 to 20 , where higher scores indicate better appetite and oral intake [32]. In present study, swallowing inactivity was defined by a SNAQ score $<14$, which has been reported to be a significant risk of weight loss $>5 \%$ within 6 months with a sensitivity of $81.5 \%$ and a specificity of $76.4 \%$ [32]. Functional performance status was determined based on self-reports using the functional recovery score (FRS) with 11 items distributed into three main areas of basic activities of daily living (4 items), instrumental activities of daily living ( 6 items), and mobility ( 1 item). Each item is rated from 0 (total dependence) to 4 (total independence) and is summarized into a weighted total score from 0 (total dependence) to 100 (complete independence) [33,34].

Nutritional status was assessed by the Mini Nutritional Assessment Short Form (MNA$\mathrm{SF}$ ) and the body mass index (BMI). The MNA-SF comprises six items related to food intake, unintentional weight loss, neuropsychological problems, acute disease, and mobility, and one item measuring BMI, which is calculated as body weight $(\mathrm{kg}) /$ squared body height $\left(\mathrm{m}^{2}\right)$ (reference range, 18.5-24.9). The MNA-SF score ranges from 0 to 14 points, where 12-14 points reflect normal nutritional status, $8-11$ points reflect risk of malnutrition, and 0-7 points reflect malnutrition [35].

Disease status was represented by degree of comorbidity burden and chronic inflammation. Comorbidity burden was determined upon the medical anamnesis of additional diagnoses (ICD-10) extracted from patients' medical charts and scored according to the age-adjusted Charlson Comorbidity Index (CCI) with a score range of 0 to 43 [36]. Chronic inflammation was represented by four inflammatory biomarkers given their previous association with sarcopenia in older adults [37,38]: C-reactive protein (CRP) (reference range $<10$ milligram per liter $(\mathrm{mg} / \mathrm{L}))$, soluble urokinase plasminogen activator receptor (suPAR) (reference range $<3$ nanogram per milliliter $(\mathrm{ng} / \mathrm{mL})$ ), tumor necrosis factor (TNF)- $\alpha$ (reference range $\leq 8.1$ picogram per $\mathrm{mL}(\mathrm{pg} / \mathrm{mL})$ ), and interleukin (IL)-6 (reference range $<16 \mathrm{pg} / \mathrm{mL}$ ) $[37,38]$. The applied measurement methods of these biomarkers are described in detail in Tavenier et al. [23].

\subsection{Statistics}

The secondary analyses were performed as complete case analyses. Initial inspections of the dataset with 125 patients who completed the EAT- 10 at baseline revealed that $48 \%$ $(n=60)$ were lost at 56-week follow-up (Supplemental Material 1, paragraph: 1.1. Loss to follow-up). In addition, there was $>20 \%$ missing values at baseline for the 30-CST and the 4MGS (Supplemental Material 1, paragraph: 1.2. Magnitude of missing values). Given that the data were not missing completely at random (Supplemental Material 1, paragraph: 1.3. Type of missingness) and that most of the variables were non-normally distributed as measured by the Kolmogorov-Smirnov test, imputation was not applied [39].

Descriptive statistics were used to summarize patient characteristics, prevalence of signs of dysphagia, and the secondary outcomes. Categorical variables were summarized as percentages, quantitative variables normally distributed as mean and standard devi- 
ation (SD), and non-normally distributed variables as median with interquartile range. Differences between patients according to swallowing status were analyzed using $t$-test for continuous data and Mann-Whitney U-test for sum-scores based on ordinal scales or continuous data non-normally distributed. For data on nominal level, continuity-corrected chi-square $\left(\chi^{2}\right)$ test or Fisher's exact test were used. The difference in dysphagia severity level across the three time points was analyzed using Friedman test. Strength of the associations between dysphagia and the parameters for probable sarcopenia as well as activity, nutritional and disease status at the three time points was also expressed as crude odds ratio (OR) with 95\% confidence intervals (95\% CI) [40]. Association of probable sarcopenia, activity status, nutrition, and disease status at baseline with signs of dysphagia (persistent and incident) at 56-week follow-up were investigated using logistic regression analysis. Due to the limited sample size and power, multinominal logistic regressions or multivariate models were not constructed [40]. All analyses were performed using the Statistical Package for Social Sciences (SPSS) version 25.0, and all $p$-values were two-sided with a significant level of $5 \%$.

\section{Results}

Table 1 displays the characteristics of 125 patients who completed the EAT-10 at baseline, the patients who completed at 56-week follow-up $(n=65)$ and those loss to follow-up $(n=60)$. Reasons for loss to follow-up are presented in Supplemental Material 1, paragraph: 1.1. Loss to follow-up. At baseline, the mean age was $78.6 \pm 8.3$ years, $56 \%$ were women, $88 \%$ lived in a private residence and about $60 \%$ were living alone. Patients who were loss to follow-up were older, had lower muscle strength and gait speed, poorer functional performance- and nutritional status, higher degree of comorbidity burden, higher plasma levels of the four inflammatory biomarkers, and longer LOS than patients who completed (Table 1). There was no difference in the EAT-10 scores between patients who completed and those lost to follow-up at 56 weeks.

Using an EAT-10 score $\geq 2$, signs of dysphagia was present in $34 \%$ of the patients at baseline, in $24 \%$ at 4 -week follow-up and in $25 \%$ at 56-week follow-up. The course of dysphagia in the subgroup of the cohort who had complete EAT-10 questionnaires at all three timepoints $(n=65)$ is illustrated in Figure 1.

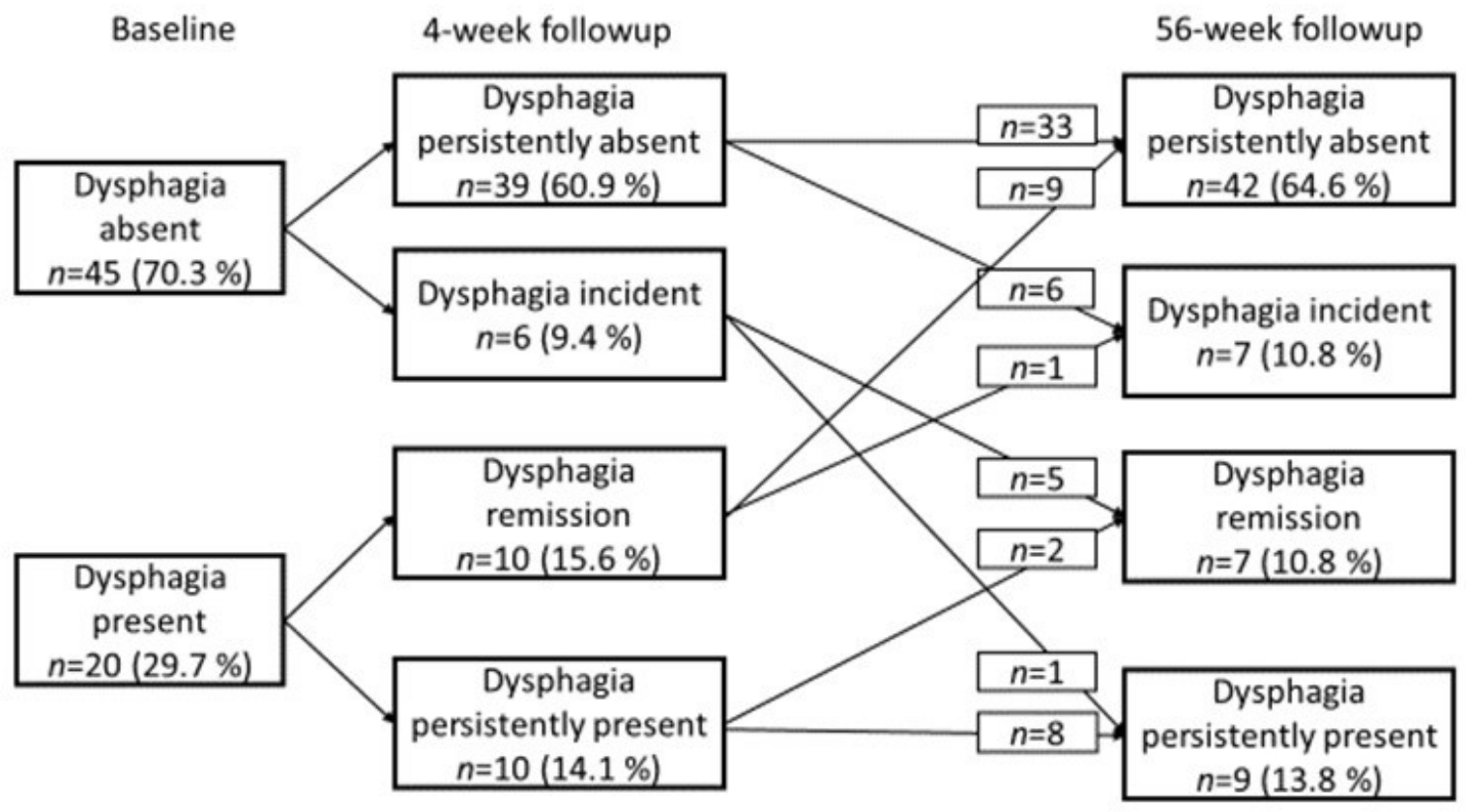

Figure 1. Course of dysphagia during a 56-weeks follow-up period. 
Table 1. Characteristics of the FAM-CPH cohort

\begin{tabular}{|c|c|c|c|c|}
\hline Variables & $\begin{array}{c}\text { Total } \\
\text { Baseline } \\
n=125\end{array}$ & $\begin{array}{c}\text { Completed } \\
56-W e e k \\
\text { Follow-Up } \\
n=65\end{array}$ & $\begin{array}{c}\text { Loss } \\
\text { 56-Week } \\
\text { Follow-Up } \\
n=60\end{array}$ & $p$ \\
\hline \multicolumn{5}{|l|}{ Demographics } \\
\hline Age in years ${ }^{a}$ & $78.6(8.3)$ & $76.7(7.8)$ & $80.7(8.4)$ & 0.007 \\
\hline Female $^{b}$ & $70(56.0 \%)$ & $35(53.8 \%)$ & $35(58.3 \%)$ & \multirow{2}{*}{0.745} \\
\hline Male $^{b}$ & $55(44.0 \%)$ & $30(46.2 \%)$ & $25(41.7 \%)$ & \\
\hline \multicolumn{5}{|l|}{ Living situation * } \\
\hline Together ${ }^{b}$ & $43(34.4 \%)$ & $24(36.9 \%)$ & $19(31.7 \%)$ & \multirow[b]{2}{*}{0.667} \\
\hline Alone ${ }^{b}$ & $82(65.6 \%)$ & $41(63.1 \%)$ & $41(68.3 \%)$ & \\
\hline \multicolumn{5}{|l|}{ Housing } \\
\hline Private residence ${ }^{b}$ & $110(88.0 \%)$ & $59(93.7 \%)$ & $51(85.0 \%)$ & \multirow{2}{*}{0.205} \\
\hline Institution ${ }^{\mathrm{b}}$ & $13(10.4 \%)$ & $4(6.3 \%)$ & $9(15.0 \%)$ & \\
\hline \multicolumn{5}{|l|}{ Cognition } \\
\hline OMC total score ${ }^{c}$ & $24(20 ; 26)$ & $24(22 ; 26)$ & $18(22 ; 26)$ & 0.064 \\
\hline \multicolumn{5}{|l|}{ Swallowing function } \\
\hline EAT-10 total score ${ }^{c}$ & $0(0 ; 3)$ & $0(0 ; 3)$ & $0(0 ; 4)$ & 0.231 \\
\hline \multicolumn{5}{|c|}{ Parameters for probable sarcopenia } \\
\hline HGS male $(\mathrm{kg})^{\text {a }}$ & $30.9(10.4)$ & $33.5(10.1)$ & $27.9(10.0)$ & 0.010 \\
\hline HGS female (kg) a & $17.7(5.9)$ & $18.9(5.7)$ & $16.5(6.0)$ & 0.098 \\
\hline $30-\mathrm{CST}^{\mathrm{c}}$ & $0(0 ; 10)$ & $8(0 ; 12)$ & $0(0 ; 5)$ & $<0.001$ \\
\hline $4 \mathrm{MGS}(\mathrm{m} / \mathrm{s})^{\mathrm{a}}$ & $0.70(0.3)$ & $0.8(0.3)$ & $0.6(0.2)$ & 0.001 \\
\hline \multicolumn{5}{|l|}{ Activity status } \\
\hline SNAQ total score ${ }^{c}$ & $14(12 ; 16)$ & $15(13 ; 16)$ & $14(12 ; 16)$ & 0.164 \\
\hline FRS total score ${ }^{c}$ & $88(77 ; 99)$ & $98(84 ; 100)$ & $84(69 ; 90)$ & $<0.001$ \\
\hline \multicolumn{5}{|l|}{ Nutritional status } \\
\hline $\operatorname{BMI}\left(\mathrm{kg} / \mathrm{m}^{2}\right)^{\mathrm{a}}$ & $26.2(5.6)$ & $26.2(4.9)$ & $26.3(6.2)$ & 0.894 \\
\hline MNA-SF total score ${ }^{c}$ & $11(8 ; 14)$ & $12(9 ; 14)$ & $10(7 ; 12)$ & 0.019 \\
\hline \multicolumn{5}{|l|}{ Reasons for admission } \\
\hline COPD exacerbation & $13(10.7 \%)$ & $\mathrm{N} / \mathrm{A}$ & $\mathrm{N} / \mathrm{A}$ & $\mathrm{N} / \mathrm{A}$ \\
\hline Dyspnea & $26(21.3 \%)$ & N/A & N/A & $\mathrm{N} / \mathrm{A}$ \\
\hline Pneumonia & $7(5.7 \%)$ & N/A & N/A & $\mathrm{N} / \mathrm{A}$ \\
\hline General symptoms \& signs & $28(23.0 \%)$ & $\mathrm{N} / \mathrm{A}$ & $\mathrm{N} / \mathrm{A}$ & $\mathrm{N} / \mathrm{A}$ \\
\hline Chest pain, unspecified & $23(18.9 \%)$ & $\mathrm{N} / \mathrm{A}$ & $\mathrm{N} / \mathrm{A}$ & $\mathrm{N} / \mathrm{A}$ \\
\hline Other causes & $25(20.5 \%)$ & N/A & N/A & N/A \\
\hline \multicolumn{5}{|l|}{ Disease status } \\
\hline $\mathrm{CCI}^{\mathrm{c}}$ & $5.0(3.0 ; 7.0)$ & $5.0(2.3 ; 6.0)$ & $4.0(6.0 ; 7.0)$ & 0.019 \\
\hline $\operatorname{CRP}(\mathrm{mg} / \mathrm{L})^{\mathrm{c}}$ & $22.0(5.1 ; 75.0)$ & $8.7(2.5 ; 45.0)$ & $48.5(3.3 ; 177.5)$ & $<0.001$ \\
\hline $\operatorname{suPAR}(\mathrm{ng} / \mathrm{mL})^{c}$ & $4.4(3.1 ; 6.2)$ & $3.3(2.8 ; 5.3)$ & $4.9(3.9 ; 6.5)$ & 0.001 \\
\hline TNF- $\alpha(\mathrm{pg} / \mathrm{mL})^{\mathrm{c}}$ & $12.5(8.4 ; 17.7)$ & $10.1(7.2 ; 13.8)$ & $15.0(10.4 ; 19.8)$ & $<0.001$ \\
\hline IL-6 $(\mathrm{pg} / \mathrm{mL})^{\mathrm{c}}$ & $4.3(1.6 ; 14.0)$ & $2.1(0.9 ; 6.3)$ & $7.8(3.2 ; 20.8)$ & $<0.001$ \\
\hline LOS (days) ${ }^{c}$ & $3.1(1.0 ; 7.2)$ & $1.8(0.8 ; 4.7)$ & $5.7(2.0 ; 9.7)$ & $<0.001$ \\
\hline
\end{tabular}

Notes: ${ }^{*}$ Institution includes nursing home, community-based rehabilitation center and senior residence. ${ }^{\text {a }}$ Mean (SD); independent $t$-test; ${ }^{\mathrm{b}} n(\%)$, Pearson $\chi^{2}$ test with continuity correction or Fischer exact test; ${ }^{\mathrm{c}}$ Median (Q1; Q3), Mann-Whitney U test. N/A, not applicable. Significant level is set at $5 \%$.

At 4-week-follow-up, signs of dysphagia were persistently absent in 39 patients (61\%), remitted in 10 patients (15\%), were incident in 6 patients $(9 \%)$, and were persistently present in 10 patients (15\%). At 56-week follow-up, signs of dysphagia were persistently absent in 42 patients $(64 \%)$, remitted in 7 patients $(11 \%)$, were incident in 7 patients $(11 \%)$ and were persistently present in 9 patients $(14 \%)$. There were no significant differences in the dysphagia severity level $\left(\chi^{2}(2)=129, p=0.524\right)$ across the three time points.

As illustrated in Table 2, signs of dysphagia were related to low HGS at baseline $(p=0.012)$ and at 56-week follow-up $(p=0.043)$, low 30-CST at baseline $(p=0.046)$ and at 4 -week $(p=0.024)$ and 56-week $(p=0.002)$ follow-up, and low 4MGS at 56-week follow-up 
$(p=0.021)$. Table 3 shows that the likelihood of presenting signs of dysphagia in the occurrence of probable sarcopenia was up to 3 times higher at baseline and 4-week follow up (ORs ranging from 1.38-3.56), and about 4 to 8 times higher at 56-week follow-up (ORs ranging from 3.79-8.14).

Table 2. Demographics, parameters for sarcopenia, activity, nutritional, and disease status according to signs of dysphagia by an EAT-score $\geq 2$ points at three timepoints.

\begin{tabular}{|c|c|c|c|c|c|c|c|c|c|}
\hline \multirow[b]{2}{*}{ Variables } & \multicolumn{3}{|c|}{ Dysphagia at Baseline } & \multicolumn{3}{|c|}{ Dysphagia at 4-Week Follow-Up } & \multicolumn{3}{|c|}{ Dysphagia at 56-Week Follow-Up } \\
\hline & $\begin{array}{c}\text { Absent } \\
n=83(66 \%)\end{array}$ & $\begin{array}{c}\text { Present } \\
n=42(34 \%)\end{array}$ & $p$ & $\begin{array}{c}\text { Absent } \\
n=70(76 \%)\end{array}$ & $\begin{array}{c}\text { Present } \\
N=22(24 \%)\end{array}$ & $p$ & $\begin{array}{c}\text { Absent } \\
n=49(75 \%)\end{array}$ & $\begin{array}{c}\text { Present } \\
n=16(25 \%)\end{array}$ & $p$ \\
\hline Age $^{a}$ & $78.7(8.7)$ & $78.5(7.3)$ & 0.979 & $77.9(7.9)$ & $80.1(10.1)$ & 0.399 & $76.1(7.9)$ & $78.6(7.2)$ & 0.156 \\
\hline Female $^{b}$ & $43(51.8 \%)$ & $27(64.3 \%)$ & \multirow{2}{*}{0.184} & $39(55.7 \%)$ & $12(54.5 \%)$ & \multirow{2}{*}{0.923} & $26(53.1 \%)$ & $9(56.3 \%)$ & \multirow{2}{*}{0.824} \\
\hline Male $^{b}$ & $40(48.2 \%)$ & $15(35.7 \%)$ & & $31(44.3 \%)$ & $10(45.5 \%)$ & & $23(46.9 \%)$ & $7(43.8 \%)$ & \\
\hline OMC-score $^{c}$ & $24(20 ; 26)$ & $22(19 ; 25)$ & 0.368 & $26(22 ; 28)$ & $24(20 ; 28)$ & 0.624 & $26(22 ; 28)$ & $26(20 ; 28)$ & 0.941 \\
\hline \multicolumn{10}{|c|}{$\begin{array}{c}\text { Parameters for probable } \\
\text { sarcopenia }\end{array}$} \\
\hline $\mathrm{HGS}^{\mathrm{b}} \leftrightarrow$ & $60(74.1 \%)$ & $21(51.2 \%)$ & \multirow{2}{*}{0.020} & $48(71.6 \%)$ & $11(55.0 \%)$ & \multirow{2}{*}{0.260} & $39(83.0 \%)$ & $9(56.3 \%)$ & \multirow{2}{*}{0.043} \\
\hline $\mathrm{HGS}^{\mathrm{b}} \downarrow$ & $21(25.9 \%)$ & $20(48.8 \%)$ & & $19(28.4 \%)$ & $9(45.0 \%)$ & & $8(17.0 \%)$ & $7(43.8 \%)$ & \\
\hline $30-\mathrm{CST}^{\mathrm{b}} \leftrightarrow$ & $27(42.2 \%)$ & $7(20.0 \%)$ & \multirow{2}{*}{0.029} & $46(68.7 \%)$ & $13(38.1 \%)$ & \multirow{2}{*}{0.024} & $37(78.7 \%)$ & $5(31.2 \%)$ & \multirow{2}{*}{0.002} \\
\hline $30-\mathrm{CST}^{\mathrm{b}} \downarrow$ & $37(57.8 \%)$ & $28(80.0 \%)$ & & $21(31.3 \%)$ & $8(61.9 \%)$ & & $10(21.3 \%)$ & $11(68.8 \%)$ & \\
\hline $4 \mathrm{MGS}^{\mathrm{b}} \leftrightarrow$ & $16(26.7 \%)$ & $5(20.0 \%)$ & \multirow[b]{2}{*}{0.591} & $28(58.2 \%)$ & $6(33.3 \%)$ & \multirow[b]{2}{*}{0.704} & $27(58.7 \%)$ & $3(20.0 \%)$ & \multirow{2}{*}{0.021} \\
\hline $4 \mathrm{MGS}^{\mathrm{b}} \downarrow$ & $44(73.3 \%)$ & $20(80.0 \%)$ & & $39(41.8 \%)$ & $12(66.7 \%)$ & & $19(41.3 \%)$ & $12(80.0 \%)$ & \\
\hline \multicolumn{10}{|l|}{ Activity status } \\
\hline SNAQ score $^{c}$ & $15(3 ; 16)$ & $13(11 ; 16)$ & 0.005 & $15(14 ; 16)$ & $14(13 ; 15)$ & 0.022 & $16(14 ; 16)$ & $14(12 ; 15)$ & $<0.001$ \\
\hline $\begin{array}{c}\text { Swallow } \\
\text { activity }{ }^{b} \leftrightarrow\end{array}$ & $56(69.1 \%)$ & $19(45.2 \%)$ & \multirow{2}{*}{0.012} & $53(75.7 \%)$ & $11(50.0 \%)$ & \multirow{2}{*}{0.043} & $42(89.4 \%)$ & $10(62.5 \%)$ & \multirow{2}{*}{0.024} \\
\hline $\begin{array}{l}\text { Swallow } \\
\text { activity }{ }^{b}\end{array}$ & $25(30.9 \%)$ & $23(54.8 \%)$ & & $17(24.3 \%)$ & $11(50.0 \%)$ & & $5(10.6 \%)$ & $6(37.5 \%)$ & \\
\hline FRS score ${ }^{c}$ & $92(80 ; 99)$ & $86(58 ; 92)$ & 0.005 & $94(78 ; 100)$ & $77(56 ; 99)$ & 0.035 & $98(88 ; 100)$ & $75(36 ; 90)$ & 0.001 \\
\hline \multicolumn{10}{|c|}{ Nutritional status } \\
\hline $\operatorname{BMI}\left(\mathrm{kg} / \mathrm{m}^{2}\right)^{\mathrm{a}}$ & $27.1(5.5)$ & $24.6(5.3)$ & 0.016 & $26.6(5.4)$ & $22.9(6.0)$ & 0.007 & $27.6(5.0)$ & $23.1(4.4)$ & 0.002 \\
\hline $\begin{array}{l}\text { MNA-SF } \\
\text { score }^{c}\end{array}$ & $11(9 ; 14)$ & $9(7 ; 12)$ & 0.004 & $11(9 ; 12)$ & $9(6 ; 12)$ & 0.117 & $14(12 ; 14)$ & $11(8 ; 13)$ & 0.002 \\
\hline Normal $^{b}$ & $30(37.0 \%)$ & $9(21.4 \%)$ & \multirow{3}{*}{0.009} & $30(43.5 \%)$ & $7(31.8 \%)$ & \multirow{3}{*}{0.185} & $38(79.2 \%)$ & $7(43.8 \%)$ & \multirow{3}{*}{0.012} \\
\hline At risk ${ }^{b}$ & $34(42.0 \%)$ & $13(31.0 \%)$ & & $29(42.0 \%)$ & $8(36.4 \%)$ & & $10(20.8 \%)$ & $7(43.8 \%)$ & \\
\hline Malnutrition ${ }^{\mathrm{b}}$ & $17(21.0 \%)$ & $20(47.6 \%)$ & & $10(14.5 \%)$ & $7(31.8 \%)$ & & $0(0.0 \%)$ & $2(12.4 \%)$ & \\
\hline \multicolumn{10}{|l|}{ Disease status } \\
\hline $\mathrm{CCI}^{\mathrm{c}}$ & $5.0(3.0 ; 7.0)$ & $5.0(3.0 ; 7.0)$ & 0.947 & NC & $\mathrm{NC}$ & & $\mathrm{NC}$ & $\mathrm{NC}$ & \\
\hline $\mathrm{CRP}(\mathrm{mg} / \mathrm{L})^{\mathrm{c}}$ & $23.0(3.8 ; 81.0)$ & $20.5(4.7 ; 54.0)$ & 0.967 & $3.3(1.3 ; 8.8)$ & $14.0(5.2 ; 18.5)$ & 0.547 & $1.9(1.0 ; 4.7)$ & $5.2(1.6 ; 11.3)$ & 0.128 \\
\hline $\begin{array}{c}\text { suPAR } \\
(\mathrm{ng} / \mathrm{mL})^{\mathrm{c}}\end{array}$ & $4.6(3.1 ; 6.3)$ & $4.0(3.0 ; 5.8)$ & 0.337 & $3.6(2.7 ; 5.3)$ & $4.7(3.1 ; 5.9)$ & 0.928 & $3.1(2.5 ; 3.8)$ & $3.2(2.3 ; 5.9)$ & 0.790 \\
\hline $\begin{array}{c}\text { TNF- } \alpha \\
(\mathrm{pg} / \mathrm{mL})^{\mathrm{c}}\end{array}$ & $12.0(8.2 ; 17.5)$ & $12.6(8.7 ; 17.9)$ & 0.739 & $9.8(7.7 ; 14.4)$ & $13.9(9.1 ; 17.8)$ & 0.260 & $9.1(6.9 ; 11.9)$ & $11.4(9.6 ; 14.4)$ & 0.039 \\
\hline $\operatorname{IL}-6(\mathrm{pg} / \mathrm{mL})^{\mathrm{c}}$ & $4.4(1.4 ; 14.3)$ & $3.7(2.1 ; 12.7)$ & 0.780 & $1.3(0.7 ; 2.3)$ & $4.1(1.7 ; 15.9)$ & 0.745 & $1.0(0.6 ; 1.5)$ & $1.4(0.8 ; 2.7)$ & 0.081 \\
\hline
\end{tabular}

Notes: ${ }^{a}$ Mean (SD), independent $t$-test; ${ }^{\mathrm{b}} n(\%)$, Pearson $\chi^{2}$ test with continuity correction or Fischer exact test; ${ }^{c}$ Median $(\mathrm{Q} 1 ; \mathrm{Q} 3)$,

Mann-Whitney U test; $\leftrightarrow$ ( = intact according to sarcopenia cut-off point $(9,29)$ ); $\downarrow$ ( = low according to sarcopenia cut-off point $(9,29)$ ); NC, not computed since CCI is only calculated for baseline data; significant level is set at $5 \%$.

Patients with signs of dysphagia had lower swallow activity and functional performance status at all three time points and showed poorer nutritional status at baseline and 56-week follow-up than patients without signs of dysphagia (Table 2). Table 3 displays that higher levels of swallow activity and functional performance and better nutritional status appeared protective for signs of dysphagia with ORs ranging from $0.81-0.98$ at baseline and 4-week follow-up, and from 0.56-0.96 at 56-week follow-up. Signs of dysphagia was not related to comorbidity burden or plasma levels for the four inflammatory biomarkers, except for TNF- $\alpha$ levels at 56-week follow-up, which were significantly higher in patients with signs of dysphagia than in patients without $(p=0.039)$ (Table 2).

The univariate logistic regressions displayed in Table 4, indicate that low leg muscle strength and endurance $(\mathrm{OR}=4.89, p=0.030)$, low swallowing activity $(\mathrm{OR}=5.61, p=0.005)$, poor nutritional status ( $\mathrm{OR}=4.35, p=0.023)$, and higher plasma levels of suPAR $(\mathrm{OR}=1.33$, $p=0.035)$ and IL-6 $(\mathrm{OR}=1.08, p=0.035)$ at baseline were associated with signs of dysphagia (persistent or incident) at 56-week follow-up. In addition, higher levels of swallow activity 
and functional performance and better nutritional status appeared protective for signs of dysphagia with ORs ranging from $0.68-0.95$.

Table 3. Association of signs of dysphagia with parameters for probable sarcopenia and activity, nutritional, and disease status at three time points

\begin{tabular}{|c|c|c|c|c|c|c|}
\hline \multirow[b]{2}{*}{ Parameters for probable sarcopenia } & \multicolumn{2}{|c|}{ Baseline } & \multicolumn{2}{|c|}{ 4-Week Follow-Up } & \multicolumn{2}{|c|}{56 Week Follow-Up } \\
\hline & $\begin{array}{l}\text { Crude OR } \\
(95 \% \mathrm{CI})\end{array}$ & $p$ & $\begin{array}{l}\text { Crude OR } \\
(95 \% \mathrm{CI})\end{array}$ & $p$ & $\begin{array}{l}\text { Crude OR } \\
(95 \% \mathrm{CI})\end{array}$ & $p$ \\
\hline $\begin{array}{c}\text { Low handgrip strength } \\
(\mathrm{HGS}<27 \mathrm{~kg} \text { (men) } /<16 \mathrm{~kg} \text { (women) })\end{array}$ & $2.72(1.23 ; 5.99)$ & 0.013 & $2.07(0.74 ; 5.78)$ & 0.167 & $3.79(1.09 ; 13.19)$ & 0.036 \\
\hline Low leg strength and endurance ( $30-\mathrm{CST}<9$ rises) & $2.92(1.11 ; 7.67)$ & 0.030 & $3.56(1.28 ; 9.88)$ & 0.015 & $8.14(2.29 ; 28.90)$ & 0.001 \\
\hline Low physical performance (4MGS $\leq 0.8 \mathrm{~m} / \mathrm{s}$ ) & $1.38(0.44 ; 4.32)$ & 0.578 & $1.44(0.48 ; 4.29)$ & 0.517 & $5.68(1.41 ; 22.93)$ & 0.015 \\
\hline \multicolumn{7}{|l|}{ Activity status } \\
\hline SNAQ score (swallow activity) & $0.81(0.70 ; 0.96)$ & 0.004 & $0.82(0.66 ; 1.02)$ & 0.075 & $0.56(0.39 ; 0.80)$ & 0.002 \\
\hline FRS score (Functional performance status) & $0.98(0.96 ; 0.99)$ & 0.019 & $0.98(0.96 ; 1.00)$ & 0.048 & $0.96(0.93 ; 0.99)$ & 0.002 \\
\hline \multicolumn{7}{|l|}{ Nutritional status } \\
\hline MNA-SF score & $0.82(0.71 ; 0.94)$ & 0.005 & $0.87(0.74 ; 1.01)$ & 0.072 & $0.64(0.47 ; 0.86)$ & 0.003 \\
\hline BMI $\left(\mathrm{kg} / \mathrm{m}^{2}\right)$ & $0.92(0.85 ; 0.99)$ & 0.019 & $0.87(0.79 ; 0.97)$ & 0.010 & $0.79(0.67 ; 0.93)$ & 0.005 \\
\hline \multicolumn{7}{|l|}{ Disease status } \\
\hline $\mathrm{CCI}$ & $1.00(0.87 ; 1.14)$ & 0.985 & NC & & NC & \\
\hline CRP (mg/L) & $1.00(0.99 ; 1.00)$ & 0.594 & $1.00(0.98 ; 1.03)$ & 0.792 & $1.11(0.98 ; 1.3)$ & 0.089 \\
\hline suPAR (ng/mL) & $0.90(0.76 ; 1.07)$ & 0.239 & $0.97(0.76 ; 1.24)$ & 0.796 & $1.15(0.87 ; 1.52)$ & 0.323 \\
\hline $\mathrm{TNF}-\alpha(\mathrm{pg} / \mathrm{mL})$ & $0.98(0.94 ; 1.02)$ & 0.421 & $1.01(0.95 ; 1.06)$ & 0.822 & $1.13(1.00 ; 1.28)$ & 0.057 \\
\hline IL-6 (pg/mL) & $1.00(0.99 ; 1.00)$ & 0.546 & $1.07(0.95 ; 1.19)$ & 0.274 & $1.16(0.94 ; 1.44)$ & 0.163 \\
\hline
\end{tabular}

Note: NC, not computed since CCI is only calculated for baseline data; significant level is set at $5 \%$.

Table 4. Associations of signs of dysphagia at 56-week follow-up and baseline status.

\begin{tabular}{ccc}
\hline \multicolumn{2}{c}{ Univariate Logistic Regression } & \\
& Crude OR (95\% CI) & $p$ \\
\hline Parameters for probable sarcopenia at baseline & \\
\hline Low handgrip strength (HGS $<27 \mathrm{~kg}(\mathrm{men}) /<16 \mathrm{~kg}($ women) $)$ & $2.96(0.88 ; 9.90)$ & 0.079 \\
Low leg strength and endurance $(30-\mathrm{CST}<9$ rises) & $4.82(1.16 ; 19.99)$ & 0.030 \\
Low physical performance (4MGS $\leq 0.8 \mathrm{~m} / \mathrm{s}))$ & $6.77(0.80 ; 57.5)$ & 0.080 \\
\hline Activity status at baseline & & \\
\hline Swallowing activity (SNAQ total score) & $0.71(0.55 ; 0.93)$ & 0.011 \\
Low swallowing activity (SNAQ $<14$ point) & $5.61(1.66 ; 19.90)$ & 0.005 \\
Functional performance status (FRS total score) & $0.95(0.92 ; 0.98)$ & 0.001 \\
\hline Nutritional status at baseline & \\
\hline Nutritional status (MNA-SF total score) & $0.68(0.54 ; 0.86)$ & 0.001 \\
Low Nutritional status (MNA-SF, at risk or malnourished) & $4.35(1.23 ; 15.44)$ & 0.023 \\
Nutritional status (BMI (kg/m $\left.{ }^{2}\right)$ & $0.87(0.76 ; 0.99)$ & 0.038 \\
\hline Disease status at baseline & \\
\hline CCI & $1.02(0.84 ; 1.25)$ & 0.828 \\
CRP (mg/L) & $1.01(1.00 ; 1.02)$ & 0.204 \\
SuPAR (ng/mL) & $1.33(1.02 ; 1.73)$ & 0.035 \\
TNF- $\alpha$ (pg/mL) & $1.12(1.00 ; 1.26)$ & 0.060 \\
IL-6 (pg/mL) & $1.08(1.01 ; 1.15)$ & 0.035 \\
\hline
\end{tabular}

Note: Significant level is set at $5 \%$.

\section{Discussion}

The present study investigated the prevalence and time course of signs of dysphagia as well as its association with parameters for probable sarcopenia, activity, nutritional status, and disease status in older patients acutely admitted to an ED. It was found that signs of dysphagia were present in $34 \%$ of the patients at baseline, in $24 \%$ at 4 -week and in $25 \%$ at 56 -week follow-up. Patients with signs of dysphagia also demonstrated signs of probable sarcopenia and they had lower swallowing activity and functional 
performance status as well as poorer nutritional status at all three time points compared to patients without signs of dysphagia. At 56-week follow-up, patients with dysphagia had higher levels of the inflammatory biomarker TNF- $\alpha$ than patients without signs of dysphagia. Univariate logistic regressions revealed that signs of probable sarcopenia as well as inactivity, malnutrition, and chronic inflammation at baseline were significantly associated with signs of dysphagia at 56-week follow-up.

The dysphagia prevalence of 34\% in the FAM-CPH-cohort at baseline is in line with the body of literature, in which cross-sectional prevalence estimates of dysphagia range from $30 \%$ to $50 \%$ in hospitalized geriatric patients [1,5-7] and in $24 \%$ of ED patients [21] However, there are large variations in the methodology for identifying dysphagia across studies. In the FAM-CPH study, EAT-10 was the only measure of dysphagia. Formal diagnosis of dysphagia is usually carried out using a comprehensive clinical bedside examination that includes physical examination of oral and motor function, and assessment of functional oral intake level of foods and liquids, or by instrumental assessments such as video fluoroscopic swallowing examination or fiberoptic endoscopic evaluation of swallowing [41]. EAT-10 is not designed for diagnosing dysphagia per se [26]. Recent work has shown that when EAT-10 is used for identifying dysphagia in older adults, it presents with low reliability and a substantially high floor effect (i.e., no problem) and might not identify patients with milder signs of dysphagia [42]. Therefore, it cannot be excluded that the prevalence of signs of dysphagia in the FAM-CPH cohort is underestimated and the course of dysphagia is biased.

This study found that signs of dysphagia in older ED patients were associated with low muscle strength and low physical performance. However, these sarcopenia-related parameters appeared more affected at baseline than at 4-week follow-up, which might be due to the patients' acute condition rather than a true reflection of muscle strength and physical performance at baseline [18]. In addition, it is particularly important to note that the data for this secondary analysis did not allow a definite diagnosis of sarcopenic dysphagia, which is defined as dysphagia due to sarcopenia in both generalized skeletal muscles and swallowing-related muscles $[8,11]$. Studies report that older patients with dysphagia and sarcopenia show low strength in tongue and lip muscles [43] and hyoid muscles [44] as well as weak pharyngeal contraction and upper esophagus sphincter dysfunction [45]. For a definite diagnosis of sarcopenic dysphagia, it is recommended to use explicit diagnostic criteria that includes the presence of dysphagia, low skeletal muscle strength and muscle mass, no obvious causative diseases of dysphagia, and low strength and mass of the swallowing muscles [8,11].

In this study, the degree of comorbidity burden according to the age adjusted CCI was relatively high and was not related to signs of dysphagia. Melgaard et al. [7] and Mañas-Martínez et al. [45] reached similar results, whereas Olesen et al. [6] and da Silva et al. [46] found that the presence of dysphagia was significantly related to higher scores of CCI. However, comorbidity is a complex concept, and it has been found that the CCI is only modestly associated with physical function [47]. Very few studies have considered the relationship between dysphagia and the state of chronic inflammation. Homem et al. [48] found a significant difference between patients with and without dysphagia regarding CRP, but not IL- 6 and TNF- $\alpha$ levels. In the present study, elevated levels of four inflammatory biomarkers were present in the whole sample. However, at 56-week follow-up, the level of TNF- $\alpha$ was significantly higher in the group of patients with signs of dysphagia. In addition, signs of dysphagia at 56-week were associated with increased baseline levels of suPAR and IL-6. Since the level of comorbidity and inflammatory biomarkers was relatively high in the FAM-CPH cohort and there was a strong and significant association of signs of dysphagia and the three sarcopenia-related parameters at 56-week follow-up, it cannot be excluded that the presence of signs of dysphagia at 56-week follow-up was influenced by sarcopenia. Chronic inflammation affects the aging body with multiple impairments, for example hormonal and/or epigenetic alterations, microvascular changes, or insulin dysregulation, which may coalesce promoting sarcopenia [49]. In a cohort of patients with 
type 2 diabetes, Kaji et al. [50] observed that whole body sarcopenia was present in about $12 \%$ and was associated with decreased tongue strength, which might indicate that the condition of decreased insulin sensitivity led to low tongue strength. However, the biology of sarcopenia is complex and involves an intricate relationship between several pro- and anti-inflammatory proteins [49]. Moreover, the complex relationship between dysphagia and sarcopenia, both primary and secondary, and how sarcopenic dysphagia should be diagnosed remains unsolved, and further research is needed $[8,11]$.

The present study found no association between signs of dysphagia and age or cognitive function, in contrasts to what has been demonstrated in previous research $[7,15]$. Since the FAM-CPH study excluded patients with severe cognitive impairments and patients who were loss to follow-up were significantly older, it cannot be excluded that cognitive function and age would have influenced the dysphagia status. Consistent with a growing body of evidence from various settings $[1,5-7,12,13,18,43,51]$, signs of dysphagia in older patients were related to reduced swallowing activity, functional performance, and nutritional status. This persisted over time after discharge from the ED department. Unfortunately, the FAM-CPH data did not include information on patients' rehabilitation plan. It has been found that in older patients with whole body sarcopenia undergoing rehabilitation for orthopedic diseases/conditions, adequate energy, and protein intake were associated with increased swallowing function and tongue strength [52]. Dysphagia and sarcopenia are both geriatric syndromes that isolated and in cooccurrence have a significant impact on older adults, resulting in poor outcomes $[1,8,11,17,53]$. The results from the FAM-CPH cohort provides support for the importance of systematic identification of sarcopenic dysphagia in at risk populations $[1,8,11,17]$ such as older patients acutely admitted to an ED department. Timely identification of dysphagia would enable initiation of early and appropriate multidisciplinary rehabilitation strategies with nutritional care, strength training of the swallowing musculature, early mobilization, and physical exercise to improve or prevent the development of sarcopenic dysphagia in this patient cohort $[8,11]$. In addition, such an approach will provide detailed information for the rehabilitation plans across health care sectors ensuring quality and efficiency in the process for the acute patient. This study is not without limitations. Application of secondary analysis on an existing dataset had some disadvantages as the data were not collected for addressing the aim of this study per se, and some study specific variables may have been left out [54]. Studies report that hospitalization may accelerate the development of sarcopenic dysphagia $[11,13]$. In present secondary analysis, patients who completed at 56-week follow-up had significantly lower LOS than patients lost to followup; and a post hoc analysis revealed that there were no significant differences in LOS between patients with or without signs of dysphagia at all three time-points. However, to determine the development of sarcopenic dysphagia after an ED-admission, a larger cohort without dysphagia at baseline would be needed. Besides, more comprehensive, and objective information on swallowing function, sarcopenia, as well as number and LOS of readmissions are required. The dataset used in present secondary analysis contained a relatively high magnitude of missing values. At 56-week follow-up, 48\% $(n=60)$ were loss and there was $>20 \%$ missing values for the 30 -CST and 4 MGS at baseline. Accordingly, the power of the statistical analyses and the validity of the results may be limited. Additionally, it was not possible to investigate whether the course of signs of dysphagia were influenced by rehabilitation strategies since the available dataset did not provide information on the patients' rehabilitation plans after the EDvisit. A significant limitation of present secondary analysis was that information on patients' muscle mass and quality was not collected as part of the FAM-CPH study. Therefore, it was only possible to confirm the presence of probable sarcopenia and not a definite diagnosis of sarcopenia, according to the EWGSOP2 [9]. This also limited a diagnosis of sarcopenic dysphagia according to suggested diagnostic criteria $[8,11]$. Thus, further investigation in a larger sample with measures of all sarcopenia-related parameters (i.e., muscle strength, muscle quantity or quality and physical performance), 
comprehensive clinical examination of dysphagia (i.e., assessment of oral and motor function and functional oral intake) and identification of possible activity-, nutritional-, and disease-related risk factors for concurrent sarcopenia and dysphagia is required to fully understand the effects of sarcopenia on the development and/or progression of dysphagia in older patients acutely admitted to an ED.

\section{Conclusions}

Signs of dysphagia are prevalent in older patients acutely admitted to the ED, persists after discharge, and are associated with probable sarcopenia, inactivity, malnutrition, and chronic inflammation, both temporary and persistently over time. Sarcopenia-related parameters such as low muscle strength and physical performance as well as inactivity of swallowing, low functional performance status and malnutrition may be potentially modifiable targets for the improvement or prevention of dysphagia in older patients upon admission and after discharge from the ED. However, additional studies on sarcopenic dysphagia in older ED patients are warranted to establish definitive prevalence estimates, time course, and predictors.

Supplementary Materials: The following are available online at https:/ /www.mdpi.com/article/10 .3390/geriatrics6020046/s1, Supplemental material 1, Paragraph S1.1: Lost to follow-up; Paragraph: 1.2: Magnitude of missing values; Paragraph S1.3: Type of missingness.

Author Contributions: Conceptualization of the secondary analysis, T.H., R.L.N. and A.L.A.; Methodology, T.H.; Software, T.H. and A.L.A.; Validation, T.H. and A.L.A.; Formal analysis, T.H.; Investigation, A.L.A., C.T., M.B.H., J.T., L.J.H.R., L.M.J., M.M.P., O.A. and R.L.N.; Resources, A.L.A., C.T., M.B.H., J.T., L.J.H.R., L.M.J., M.M.P., O.A. and R.L.N.; Data curation, A.L.A. and O.A.; Writingoriginal draft preparation, T.H.; Writing—review and editing, A.M.B., A.L.A., C.T., M.B.H., J.T., J.P., L.J.H.R., L.M.J., M.M.P., O.A. and R.L.N.; Visualization, T.H.; Project administration, A.L.A. and O.A.; Funding acquisition, A.L.A., M.B.H., J.T., J.P., L.J.H.R. and O.A. All authors have read and agreed to the published version of the manuscript.

Funding: This secondary analysis received no external funding. The FAM-CPH study was supported by Laege Sofus Carl Emil Friis og Hustru Olga Doris Friis' Legat; the Toyota Foundation (grant number KJ/BG-9305 F to O.A.); the A.P. Moeller Foundation for the Advancement of Medical Science (grant number 17-L-0291 to L.J.H.R.); the Research Foundation of Hvidovre Hospital; and the Lundbeck Foundation (grant numbers R180-2014-3360, R288-2018-380 to L.J.H.R.).

Institutional Review Board Statement: The FAM-CPH study was conducted according to the guidelines of the Declaration of Helsinki, and the study protocol was approved by the Danish Data Protection Agency (AHH-2016-067) and the Research Ethics Committees for the Capital Region of Denmark (H-16038786).

Informed Consent Statement: Informed consent was obtained from all subjects involved in the study.

Data Availability Statement: Data available on request due to restrictions. The data presented in this study are not publicly available due to Danish legislation. Request to access the dataset will require an individual inquiry to the Danish Data Protection agency for approval.

Acknowledgments: The data used for this secondary analysis were collected as part of the FAM-CPH study [22,23] performed as a part of the acute-CAG (Clinical Academic Group-REcoveryCAPacity) nominated by Greater Copenhagen Health Science Partners (GCHSP). The data were used with permission of the acute-CAG.

Conflicts of Interest: The authors declare no conflict of interest.

\section{References}

1. Baijens, L.W.; Clavé, P.; Cras, P.; Ekberg, O.; Forster, A.; Kolb, G.F.; Leners, J.C.; Masiero, S.; Mateos-Nozal, J.; Ortega, O.; et al. European Society for Swallowing Disorders-European Union Geriatric Medicine Society white paper: Oropharyngeal dysphagia as a geriatric syndrome. Clin. Interv. Aging. 2016, 7, 1403-1428. [CrossRef]

2. Miyashita, T.; Kikutani, T.; Nagashima, K.; Igarashi, K.; Tamura, F. The effects of sarcopenic dysphagia on the dynamics of swallowing organs observed on videofluoroscopic swallowing studies. J. Oral. Rehabil. 2020, 47, 584-590. [CrossRef] 
3. Swan, K.; Speyer, R.; Heijnen, B.J.; Wagg, B.; Cordier, R. Living with oropharyngeal dysphagia: Effects of bolus modification on health-related quality of life-a systematic review. Qual. Life Res. 2015, 24, 2447-2456. [CrossRef]

4. Verdonschot, R.J.; Baijens, L.W.; Vanbelle, S.; van de Kolk, I.; Kremer, B.; Leue, C. Affective symptoms in patients with oropharyngeal dysphagia: A systematic review. J. Psychosom. Res. 2017, 97, 102-110. [CrossRef]

5. Westmark, S.; Melgaard, D.; Rethmeier, L.O.; Ehlers, L.H. The cost of dysphagia in geriatric patients. Clinicoecon. Outcomes Res. 2018, 10, 321-326. [CrossRef] [PubMed]

6. Olesen, M.D.; Modlinski, R.M.; Poulsen, S.H.; Rosenvinge, P.M.; Rasmussen, H.H.; Holst, M. Prevalence of signs of dysphagia and associated risk factors in geriatric patients admitted to an acute medical unit. Clin. Nutr. ESPEN 2021, 41, 208-216. [CrossRef]

7. Melgaard, D.; Rodrigo-Domingo, M.; Mørch, M.M. The Prevalence of Oropharyngeal Dysphagia in Acute Geriatric Patients. Geriatrics 2018, 3, 15. [CrossRef] [PubMed]

8. Fujishima, I.; Fujiu-Kurachi, M.; Arai, H.; Hyodo, M.; Kagaya, H.; Maeda, K.; Mori, T.; Nishioka, S.; Oshima, F.; Ogawa, S.; et al. Sarcopenia and dysphagia: Position paper by four professional organizations. Geriatr. Gerontol. Int. 2019, $19,91-97$. [CrossRef] [PubMed]

9. Cruz-Jentoft, A.J.; Bahat, G.; Bauer, J.; Boirie, Y.; Bruyère, O.; Cederholm, T.; Cooper, C.; Landi, F.; Rolland, Y.; Sayer, A.A.; et al. Writing Group for the European Working Group on Sarcopenia in Older People 2 (EWGSOP2), and the Extended Group for EWGSOP2. Sarcopenia: Revised European consensus on definition and diagnosis. Age Ageing 2019, 48, 16-31. [CrossRef] [PubMed]

10. Zhao, W.T.; Yang, M.; Wu, H.M.; Yang, L.; Zhang, X.; Huang, Y. Systematic Review and Meta-Analysis of the Association between Sarcopenia and Dysphagia. J. Nutr. Health Aging 2018, 22, 1003-1009. [CrossRef]

11. Wakabayashi, H.; Kishima, M.; Itoda, M.; Fujishima, I.; Kunieda, K.; Ohno, T.; Shigematsu, T.; Oshima, F.; Mori, T.; Ogawa, N.; et al. Working Group on Sarcopenic Dysphagia. Diagnosis and Treatment of Sarcopenic Dysphagia: A Scoping Review. Dysphagia 2021. [CrossRef] [PubMed]

12. Cha, S.; Kim, W.S.; Kim, K.W.; Han, J.W.; Jang, H.C.; Lim, S.; Paik, N.J. Sarcopenia is an Independent Risk Factor for Dysphagia in Community-Dwelling Older Adults. Dysphagia 2019, 34, 692-697. [CrossRef] [PubMed]

13. Maeda, K.; Akagi, J. Sarcopenia is an independent risk factor of dysphagia in hospitalized older people. Geriatr. Gerontol. Int. 2016, 16, 515-521. [CrossRef]

14. Nagano, A.; Maeda, K.; Shimizu, A.; Nagami, S.; Takigawa, N.; Ueshima, J.; Suenaga, M. Association of Sarcopenic Dysphagia with Underlying Sarcopenia Following Hip Fracture Surgery in Older Women. Nutrients 2020, 12, 1365. [CrossRef] [PubMed]

15. Firat Ozer, F.; Akın, S.; Soysal, T.; Gokcekuyu, B.M.; Erturk Zararsız, G. Relationship Between Dysphagia and Sarcopenia with Comprehensive Geriatric Evaluation. Dysphagia 2021, 36, 140-146. [CrossRef] [PubMed]

16. Wakabayashi, H.; Takahashi, R.; Murakami, T. The prevalence and prognosis of sarcopenic dysphagia in patients who require dysphagia rehabilitation. J. Nutr. Health Aging 2019, 23, 84-88. [CrossRef]

17. Nagano, A.; Nishioka, S.; Wakabayashi, H. Rehabilitation nutrition for iatrogenic sarcopenia and sarcopenic dysphagia. J. Nutr. Health Aging 2019, 23, 265. [CrossRef]

18. Maeda, K.; Ishida, Y.; Nonogaki, T.; Shimizu, A.; Yamanaka, Y.; Matsuyama, R.; Kato, R.; Mori, N. Development and Predictors of Sarcopenic Dysphagia during Hospitalization of Older Adults. Nutrients 2019, 12, 70. [CrossRef]

19. Fløjstrup, M.; Bogh, S.B.; Henriksen, D.P.; Bech, M.; Johnsen, S.P.; Brabrand, M. Increasing emergency hospital activity in Denmark, 2005-2016: A nationwide descriptive study. BMJ Open 2020, 10, e031409. [CrossRef]

20. Hald, A.N.; Bech, M.; Burau, V. Conditions for successful interprofessional collaboration in integrated care-Lessons from a primary care setting in Denmark. Health Policy 2021, 125, 474-481. [CrossRef]

21. Melgaard, D.; Sorensen, L.R.; Lund, D.; Leutscher, P.; Ludwig, M. Systematic Dysphagia Screening of Elderly Persons in the Emergency Department-A Feasibility Study. Geriatrics 2020, 5, 75. [CrossRef]

22. Houlind, M.B.; Andersen, A.L.; Treldal, C.; Jørgensen, L.M.; Kannegaard, P.N.; Castillo, L.S.; Christensen, L.D.; Tavenier, J.; Rasmussen, L.J.H.; Ankarfeldt, M.Z.; et al. A collaborative medication review including deprescribing for older patients in an Emergency Department: A longitudinal feasibility study. J. Clin. Med. 2020, 9, 348. [CrossRef]

23. Tavenier, J.; Rasmussen, L.J.H.; Andersen, A.L.; Houlind, M.B.; Langkilde, A.; Andersen, O.; Petersen, J.; Nehlin, J.O. Association of GDF15 with inflammation and physical function during aging and recovery after acute hospitalization: A longitudinal study of older patients and age-matched controls. J. Gerontol. A Biol. Sci. Med. Sci. 2021, glab011. [CrossRef] [PubMed]

24. Harris, P.A.; Taylor, R.; Minor, B.L.; Elliott, V.; Fernandez, M.; O’Neal, L.; McLeod, L.; Delacqua, G.; Delacqua, F.; Kirby, J.; et al. The REDCap Consortium: Building an international community of software platform partners. J. Biomed. Inform. 2019, 95, 103208. [CrossRef] [PubMed]

25. Wade, D.T.; Vergis, E. The Short Orientation-Memory-Concentration Test: A study of its reliability and validity. Clin. Rehab 1999, 13, 164-170. [CrossRef]

26. Belafsky, P.C.; Mouadeb, D.A.; Rees, C.J.; Pryor, J.C.; Postma, G.N.; Allen, J.; Leonard, R.J. Validity and reliability of the eating assessment tool (EAT-10). Ann. Otol. Rhinol. Laryngol. 2008, 117, 919-924. [CrossRef]

27. Rofes, L.; Arreola, V.; Mukherjee, R.; Clave, P. Sensitivity and specificity of the eating assessment tool and the volumeviscosity swallow test for clinical evaluation of oropharyngeal dysphagia. Neuro Gastroenterol. Motil. 2014, $26,1256 \mathrm{e} 65$. [CrossRef] [PubMed] 
28. Lee, S.H.; Gong, H.S. Measurement and Interpretation of Handgrip Strength for Research on Sarcopenia and Osteoporosis. J. Bone Metab. 2020, 27, 85-96. [CrossRef]

29. Jones, C.; Rikli, R.E.; Beam, W.C. A 30-s chair-stand test as a measure of lower body strength in community-residing older adults. Res. Q. Exerc. Sport 1999, 70, 113-119. [CrossRef] [PubMed]

30. Bohannon, R.W.; Wang, Y.C. Four-Meter Gait Speed: Normative Values and Reliability Determined for Adults Participating in the NIH Toolbox Study. Arch. Phys. Med. Rehabil. 2019, 100, 509-513. [CrossRef] [PubMed]

31. Bruun, I.H.; Mogensen, C.B.; Norgaard, B.; Schiøttz-Christensen, B.; Maribo, T. Validity and Responsiveness to Change of the 30-Second Chair-Stand Test in Older Adults Admitted to an Emergency Department. J. Geriatr Phys. Ther. 2019, 42, 265-274. [CrossRef] [PubMed]

32. Wilson, M.M.; Thomas, D.R.; Rubenstein, L.Z.; Chibnall, J.T.; Anderson, S.; Baxi, A.; Diebold, M.R.; Morley, J.E. Appetite assessment: Simple appetite questionnaire predicts weight loss in community-dwelling adults and nursing home residents. Am. J. Clin. Nutr. 2005, 82, 1074-1081. [CrossRef]

33. Zuckerman, J.D.; Koval, K.J.; Aharonoff, G.B.; Skovron, M.L. A functional recovery score for elderly hip fracture patients: I. Development. J. Orthop Trauma 2000, 14, 20-25. [CrossRef]

34. Zuckerman, J.D.; Koval, K.J.; Aharonoff, G.B.; Skovron, M.L. A functional recovery score for elderly hip fracture patients: II. Validity and reliability. J. Orthop. Trauma 2000, 14, 26-30. [CrossRef] [PubMed]

35. Kaiser, M.J.; Bauer, J.M.; Ramsch, C.; Uter, W.; Guigoz, Y.; Cederholm, T.; Thomas, D.R.; Anthony, P.; Charlton, K.E.; Maggio, M.; et al. Validation of the Mini Nutritional Assessment short-form (MNA-SF): A practical tool for identification of nutritional status. J. Nutr. Health Aging 2009, 13, 782-788. [CrossRef] [PubMed]

36. Hall, W.H.; Ramachandran, R.; Narayan, S.; Jani, A.B.; Vijayakumar, S. An electronic application for rapidly calculating Charlson comorbidity score. BMC Cancer 2004, 20, 94. [CrossRef]

37. Wyczalkowska-Tomasik, A.; Czarkowska-Paczek, B.; Zielenkiewicz, M.; Paczek, L. Inflammatory Markers Change with Age, but do not Fall Beyond Reported Normal Ranges. Arch. Immunol. Ther. Exp. (Warsz) 2016, 64, 249-254. [CrossRef]

38. Pisinger, C.; Eugen-Olsen, J. Risk factors associated with serum levels of the inflammatory biomarker soluble urokinase plasminogen activator receptor in a general population. Biomark. Insights 2014, 9, 91-100. [CrossRef]

39. Lodder, P. To impute or not impute, that's the question. In Advising on Research Methods: Selected Topics; Mellenbergh, J.G., Adèr, H.J., Eds.; Johannes van Kessel Publishing: Huizen, The Netherlands, 2013.

40. Altman, D.G. Practical Statistics for Medical Research; CRC Press: Boca Raton, FL, USA; Chapman and Hall: London, UK, 1991.

41. Shaker, R.; Easterling, C.; Belafsky, P.C.; Postma, G.N. (Eds.) Manual of Diagnostic and Therapeutic Techniques for Disorders of Deglutition; Springer Science \& Business Media: New York, NY, USA, 2012.

42. Hansen, T.; Kjaersgaard, A. Item analysis of the Eating Assessment Tool (EAT-10) by the Rasch model: A secondary analysis of cross-sectional survey data obtained among community-dwelling elders. Health Qual. Life Outcomes 2020, 18, 139. [CrossRef]

43. Sakai, K.; Nakayama, E.; Tohara, H.; Kodama, K.; Takehisa, T.; Takehisa, Y.; Ueda, K. Relationship between tongue strength, lip strength, and nutrition-related sarcopenia in older rehabilitation inpatients: A cross-sectional study. Clin. Interv. Aging. 2017, 12, 1207-1214. [CrossRef]

44. Chen, Y.C.; Chen, P.Y.; Wang, Y.C.; Wang, T.G.; Han, D.S. Decreased swallowing function in the sarcopenic elderly without clinical dysphagia: A cross-sectional study. BMC Geriatr. 2020, 20, 419. [CrossRef]

45. Mañas-Martínez, A.B.; Bucar-Barjud, M.; Campos-Fernández, J.; Gimeno-Orna, J.A.; Pérez-Calvo, J.; Ocón-Bretón, J. Association of positive screening for dysphagia with nutritional status and long-term mortality in hospitalized elderly patients. Endocrinol. Diabetes. Nutr. 2018, 65, 402-408. [CrossRef]

46. Da Silva, A.F.; Moreira, E.A.M.; Barni, G.C.; Panza, V.S.P.; Furkim, A.M.; Moreno, Y.M.F. Relationships between high comorbidity index and nutritional parameters in patients with Oropharyngeal Dysphagia. Clin. Nutr. ESPEN 2020, 38, 218-222. [CrossRef]

47. Kabboord, A.D.; van Eijk, M.; Fiocco, M.; van Balen, R.; Achterberg, W.P. Assessment of Comorbidity Burden and its Association with Functional Rehabilitation Outcome after Stroke or Hip Fracture: A Systematic Review and Meta-Analysis. J. Am. Med. Dir. Assoc. 2016, 17, 1066.e13-1066.e21. [CrossRef]

48. Homem, S.G.; Moreira, E.A.M.; da Silva, A.F.; Barni, G.C.; da Rosa, J.S.; Oliveira, D.L.; Tomio, C.; de Freitas, M.B.; Portari, G.V.; Furkim, A.M.; et al. Relationship between oropharyngeal dysphagia, nutritional status, antioxidant vitamins and the inflammatory response in adults and elderly: A cross-sectional study. Clin. Nutr. ESPEN 2020, 38, 211-217. [CrossRef]

49. Chhetri, J.K.; de Souto Barreto, P.; Fougère, B.; Rolland, Y.; Vellas, B.; Cesari, M. Chronic inflammation and sarcopenia: A regenerative cell therapy perspective. Exp. Gerontol. 2018, 103, 115-123. [CrossRef] [PubMed]

50. Kaji, A.; Hashimoto, Y.; Kobayashi, Y.; Sakai, R.; Okamura, T.; Miki, A.; Hamaguchi, M.; Kuwahata, M.; Yamazaki, M.; Fukui, M. Sarcopenia is associated with tongue pressure in older patients with type 2 diabetes: A cross-sectional study of the KAMOGAWADM cohort study. Geriatr Gerontol Int. 2019, 19, 153-158. [CrossRef]

51. Yoshimura, Y.; Wakabayashi, H.; Bise, T.; Tanoue, M. Prevalence of sarcopenia and its association with activities of daily living and dysphagia in convalescent rehabilitation ward inpatients. Clin. Nutr. 2018, 37, 2022-2028. [CrossRef] [PubMed]

52. Nagano, A.; Maeda, K.; Koike, M.; Murotani, K.; Ueshima, J.; Shimizu, A.; Inoue, T.; Sato, K.; Suenaga, M.; Ishida, Y.; et al. Effects of physical rehabilitation and nutritional intake management on improvement in tongue strength in sarcopenic patients. Nutrients 2020, 12, 3104. [CrossRef] 
53. Yeung, S.S.Y.; Reijnierse, E.M.; Pham, V.K.; Trappenburg, M.C.; Lim, W.K.; Meskers, C.G.M.; Maier, A.B. Sarcopenia and its association with falls and fractures in older adults: A systematic review and meta-analysis. J. Cachexia Sarcopenia Muscle 2019, 10, 485-500. [CrossRef] [PubMed]

54. Cheng, H.G.; Phillips, M.R. Secondary analysis of existing data: Opportunities and implementation. Shanghai Arch. Psychiatry 2014, 26, 371-375. [CrossRef] [PubMed] 